\title{
Image design and promotion of city bus station -- Taking Qinhuangdao city as an example
}

\author{
Chao ZHAO ${ }^{1, a^{*}}$, Wei-Xi ZHANG ${ }^{1, b}$ and Dong ZENG ${ }^{2, c}$ \\ ${ }^{1}$ College of Art and Design, Yanshan University, Qinhuangdao, Hebei, China \\ ${ }^{2}$ Hebei Normal University of Science and Technology, Qinhuangdao, Hebei, China \\ azhaochao_616@126.com, b269290989@qq.com, c260378069@qq.com \\ ${ }^{*}$ Corresponding author
}

Keywords: City image, bus stop, design, humanized layout.

\begin{abstract}
The bus station in Qinhuangdao city was re design, is to transfer ahumanized design concept, humanized design is the process of human transformation of the world has been seeking, is the highest stage of design development. The project to "city bus stop design"as the main line, to shape the city image by image design andplanning schedule, use what they have learned, see, to find the characteristics of the city of Qinhuangdao culture and brand charm.To further enrich the entity of the original image, but also highlight the innovative experiment project. The city street design and visualculture will play a guiding role model.
\end{abstract}

\section{Foreword}

Through a large number of empirical research on Qinhuangdao city static bus stop, discrete analysis through survey data, the application of psychological principles to static bus stop problem classification to physiological and safety, behavior and psychological, cultural and emotional three aspects of demand, starting from the optimization of stop, the prospect of a high performance, high quality public transport system city, city traffic structure optimization, the growing traffic problems be solved effectively. Such a public transport developed city, is a harmonious city economy society, adhere to the road of sustainable development.

\section{Background, conception}

The more frequent city residents, floating population, students, retired elderly people in the post's social intercourse, especially Qinhuangdao large group of students, the public traffic demand rising.

\section{The importance of the 1 public transportation}

So in an economical society background, take the sustainable development as the premise, guarantee the superiority and efficiency of public transport, vigorously develop the city public transportation, optimize the structure of city traffic, is the effective measure to solve the contradiction between supply and demand of city traffic is increasing.

An integral part of the bus stop as a city plays an important role in the whole city visual image. Qinhuangdao as a national well-known tourist city, the perfect visual image will makes the whole city to add a beautiful color. Qinhuangdao as a national well-known tourist city, the perfect visual image will makes the whole city to add a beautiful color. Therefore, the effective ways to improve the city cultural taste, design quality is achieved through the humanized design to improve the bus station.

We adopted the Qinhuangdao public investigation and related literatures we know, bus stop in Qinhuangdao is not accepted by the public, the re design is particularly important. 


\section{The prominent problem of 2 public transportation bus stop comprehensive problems}

Bus stop is more passengers stay in place, and the bus station is the first interface tool contact passengers and the bus, bus stop bus station carrying a passenger gets on the train information, wait for duties, is a powerful media, the problem is the general public concern and the hope of bus system effectively improve.

\section{Design objective}

The bus stop of a city is an important part of city public transportation system, is an important index to evaluate a city civilization degree and the level of economic development, the bus schedule changes will improveQinhuangdao's image, transit service quality promotion of Qinhuangdao City, to help people better travel. At the same time also has certain help to the vulnerable groups, give a care and help for them, so that they can better enjoy life, enjoy the convenience and convenient design.

The bus stop is a kind of distributed in various public transportation line site, providing public transport vehicles, to convey information or long-term, or temporary physical media to the public transportation passenger. Bus routes in Qinhuangdao city is relatively less, but because of the economic level is limited, if we want to realize the bus electronic information display system is not the reality. While the static bus stop, whether at home or abroad has a long history, and is still used by most of the world, is the main part of the media to convey the road traffic information. The relative advantage obviously low manufacturing cost, convenient maintenance and management, which covers an area of small, easy to be accepted by people.

The bus station is the important infrastructure in a city, and the bus station is an important part of the whole city bus system. The bus station in Qinhuangdao city was made to design, in order to deliver a humanized design concept, humanized design is the process of human transformation of the world has been seeking, is the highest stage of design development, is to let the audience know that a simple bus station card also can have such beauty and so convenient practicality, let more designers to participate in such a design, let more city to accept such a design, this has certain effect to improve the level of the overall design of the China. Through this change can let more people feel the spirit of enjoyment.

\section{Positioning analysis}

The problem of static bus stop is summarized, combining scientific humanism representative Maslow (Abraham H.Maslow) analysis on the demand level, the problems are divided into three categories to analyze and solve: physiological and safety needs, behavior and psychological needs and the cultural and emotional needs three hand:

\section{Physiological and safety needs}

The bus stop is a long-standing problem, many factors need to be combined with the user, road, building, traffic, and environment and planning to consider. But the physiological need of users the most fundamental has been seriously neglected. The author analyzes Qinhuangdao people, most in need of attention is the blind, they cannot use visual information orientation, orientation to engage in activities, and need the help of other sensory understanding environment, orientation, and location of activities. The author also analyzes the relationship between the bus stop and human scale, can know the visual characteristics of human body is determined by the composition of human visual organ, therefore, to the bus station to make it more consistent with the change of various (including the visually impaired) visual characteristics is very necessary. 


\section{Behavioral and psychological needs}

Qinhuangdao city all the static bus stop, train information card provides information on the site, only the departure time, charging three kinds of train information. These have been far can not meet the more comprehensive information on the trips people want to understand the psychological needs. With the development of the times, the riding behavior of three kinds of train information basic cans no longer effectively the whole range guide public. 3 cultural and emotional needs

\section{Cultural and emotional needs}

The bus stop is belong to the people to adapt to environmental tool, and belongs to the cultural category. Bus stop from itself in addition to meet these two requirements, an important element to create a cultural environment and harmonious city, to meet people's cultural and emotional needs.

Qinhuangdao as a tourist city, but also as a historical city, full of rich humanistic. The bus stop as broadly City coverage, and daily contact with many advantages such as convenience of media, provides the perfect place to publicize the Qin culture and humanistic spirit. The bus stop is set up for people waiting for the bus, it also plays a pedestrian (especially outsiders) the role of guide, take as a tourist city logo proper function. Into some new functions, such as: for the bus stop near the landmark positions, commercial outlets range, major public facilities location (Toilet, telephone etc.), introduction to the nearby attractions (Park, temple, the square), which is bound to bring more convenience and comfort for people in the city.

\section{Application}

The empirical study of Qinhuangdao city through large amounts of static bus stop, found many Qinhuangdao static bus schedule problems. According to the research result can reach the design direction:

\section{Unified color: black and white, black and yellow}

Deep bottom expansion effect than the white rich white effect the bottom depth of word a strong, convey information faster.

\section{Unified font size}

In a normal human vision to see a number and a number of fonts is appropriate, and strictly control the text height, so as to avoid deformation caused by the apparent distance decreased.

\section{To solve the layout problem}

We found some stop using horizontal some can be vertical, so there are typeset orientation problem. We suggest that as soon as possible to standardize the font color, size and layout.

\section{On the train information bar added}

Interval: crowded bus stations every line peak interval, each line;

Transfer of information: near the line, line transfer instructions;

Near the station distance apart: add site distance between the train information site, accurate to M.

\section{The conclusion}

Although it is a small bus stop, but because of its coverage area, the majority of the large number of users, coupled with the new requirements of new era, the above problems are very much. Bus stop in Qinhuangdao city both in physical security level, behavior psychology, culture or emotional level, cannot satisfy the demand of people, three kinds of human needs are ignored in different degree. The author suggests, the relevant departments are targeted for rectification of bus stop, and 
"people-oriented" from the people's basic needs, to fully consider the various needs of the public, the bus stop is more humanized, and gradually improve the public transport system, the hardware and software can meet the needs of the general public, so that the public can regain its the basic needs of rights.

\section{References}

[1]Dongchu Zhang. Design of [J]. city of city public utilities in industrial design, 2003

[2]Chao Zhao, had winter. Talk about Chu culture "the unity of heaven and the symbol [J]. design building a city",2012 (2)

[3] Kenya Hara. Design in the design of [M]. Ji'nan: Shandong people's publishing house, 2010

[4]van .Design of behavior and cognitive humanization design [M]. Beijing: China electric power press, 2009

[5]Yi Zheng. Several aspects of [J]. decoration should be noted that the static public transport stop design, 2005(145): 10

[6] piece of nature. Beijing bus station information layout design in [J]. decoration, 2005 (145): 8

[7]Baoxiang Ruan. Ergonomics - curriculum design / courses selected papers from the [M]. Beijing: Mechanical Industry Press, 2005 (5): 105

[8]Chao Zhao. A preliminary study on the visual guide design [J]. design, 2013 (11)

[9]Minmin Guo. Art and design of personalized era [J].city public facility design (Theory), 200807

[10]Xuan Cao . Study on the city public facility humanization design [J]. Qilu art gallery, in 200803 